\title{
ISS-CREAM Flight Operation
}

\author{
K.C. Kim ${ }^{1,2, *}$, Y. Amare ${ }^{1}$, D. Angelaszek ${ }^{1,2}$, N. Anthony ${ }^{1}$, G.H. Choi ${ }^{3}$, M. Chung ${ }^{1}$, \\ M. Copley ${ }^{1}$, L. Derome ${ }^{4}$, L. Eraud ${ }^{4}$, C. Falana ${ }^{1}$, A. Gerrety ${ }^{1}$, L. Hagenau ${ }^{1}$, J.H. Han ${ }^{1}$, \\ H.G. Huh ${ }^{1}$, Y.S. Hwang ${ }^{5}$, H.J. Hyun ${ }^{5}$, H.B. Jeon ${ }^{5}$, J.A. Jeon ${ }^{3}$, S. Jeong ${ }^{3}$, S.C. Kang ${ }^{5}$,

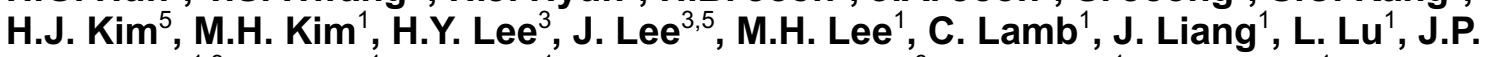 \\ Lundquist $^{1,3}$, L. Lutz ${ }^{1}$, B. Mark ${ }^{1}$, A. Mechaca-Rocha ${ }^{6}$, T. Mernik ${ }^{1}$, M. Nester ${ }^{1}, 0$. \\ Ofoha $^{1}$, H. Park ${ }^{5}$, I.H. Park ${ }^{3}$, J.M. Park ${ }^{5}$, N. Picot-Clemente ${ }^{1}$, S. Rostsky ${ }^{1}$, E.S. \\ Seo $^{1,2}$, J.R. Smith ${ }^{1}$, R. Takeishi ${ }^{3}$, T. Tatoli ${ }^{1}$, P. Walpole ${ }^{1}$, R.P. Weinmann ${ }^{1}$, J. Wu ${ }^{1}$, Z. \\ Yin $^{1,2}$, Y.S. Yoon ${ }^{1}$, H.G. Zhang ${ }^{1}$ \\ ${ }^{1}$ Inst. for Phys. Sci. and Tech., University of Maryland, College Park, MD, USA \\ ${ }^{2}$ Dept. of Physics, University of Maryland, College Park, MD, USA \\ ${ }^{3}$ Dept. of Physics, Sungkyunkwan University, Republic of Korea \\ ${ }^{4}$ Laboratoire de Physique Subatomique et de Cosmologie, Grenoble, France \\ ${ }^{5}$ Dept. of Physics, Kyungpook National University, Republic of Korea \\ ${ }^{6}$ Instituto de Fisica, Universidad Nacional Autonoma de Mexico, Mexico
}

\begin{abstract}
The Cosmic Ray Energetics And Mass experiment for the International Space Station (ISSCREAM) is designed and built to measure the elemental energy spectra of cosmic-ray particles $(1 \leq \mathrm{Z} \leq 26)$ and electrons. It measures the energy of incident cosmic rays from $10^{12}$ to $10^{15} \mathrm{eV}$. ISS-CREAM was launched and deployed to the ISS in August 2017. The Science Operations Center (SOC) at the University of Maryland has been operating the payload on the International Space Station (ISS) in coordination with the Payload Operations Integration Center (POIC) at NASA's Marshall Space Flight Center. The SOC has been responsible for sending commands to and receiving data from the Science Flight Computer (SFC) on board ISS-CREAM. The ISSCREAM data taking program interfaces with the POIC using the Telescience Resources Kit through the Software Toolkit for Ethernet Lab-Like Architecture developed by the Boeing Company. The command uplink and data downlink have been through the Tracking and Data Relay Satellite System. We present the ISS-CREAM flight operations including ISS communications, SFC performance, etc.
\end{abstract}

36th International Cosmic Ray Conference -ICRC2019-

July 24th - August 1st, 2019

Madison, WI, U.S.A.

\footnotetext{
${ }^{1}$ Speaker for the ISS-CREAM Collaboration

*E-mail: kckim@umd.edu

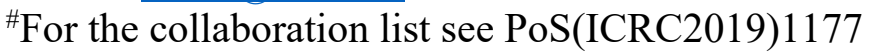




\section{Introduction}

The ISS-CREAM experiment [1] is the successor of the Antarctic balloon-borne CREAM mission [2], which flew successfully 7 times for a total accumulated time of 191 days. It was launched and installed on the Japanese Experiment Module External Facility 2 (JEM-EF 2) at the ISS in August, 2017. ISS-CREAM is aimed to measure cosmic rays to the highest energy possible up to the "so-called" knee at a few PeV. For this purpose, the ISS offers an ideal platform to increase the exposure to these particles by at least an order of magnitude compared to previous experiments. Taking data on the ISS also provides the advantage not to necessitate any atmospheric corrections. The ISS-CREAM instrument is made of multiple independent particle detectors that, combined together, can characterize incident particles. The charge of cosmic rays is measured using 4 independent layers of silicon charge detectors (SCD) $[3,4]$, sensitive in the range from $Z=1$ to $Z=26$. Their energy is obtained from the 20 -radiation length electromagnetic sampling calorimeter $(\mathrm{CAL})$, with a preceding densified graphite target that initiates hadronic interactions of cosmic-ray nuclei. The ISS-CREAM CAL is designed to measure energies from a few hundred $\mathrm{GeV}$ to $1 \mathrm{PeV}$. Electron/proton separation can be performed with the CAL, the top and bottom counting detectors (T/BCD) [5, 6], and the boronated scintillator detector (BSD) [7]. Triggering of incident particles is performed by using the CAL and T/BCD. Consecutive layers of the CAL comprised of a tungsten plate plus scintillating fibers are interleaved with epoxycoated fiberglass to comply with space launch requirements. In order to characterize and verify the performance of the detector in this configuration, beam tests were performed at the CERNSPS in 2015 [8]. The overall configuraiton is shown in Figure 1. This paper presents the ISSCREAM flight operation.

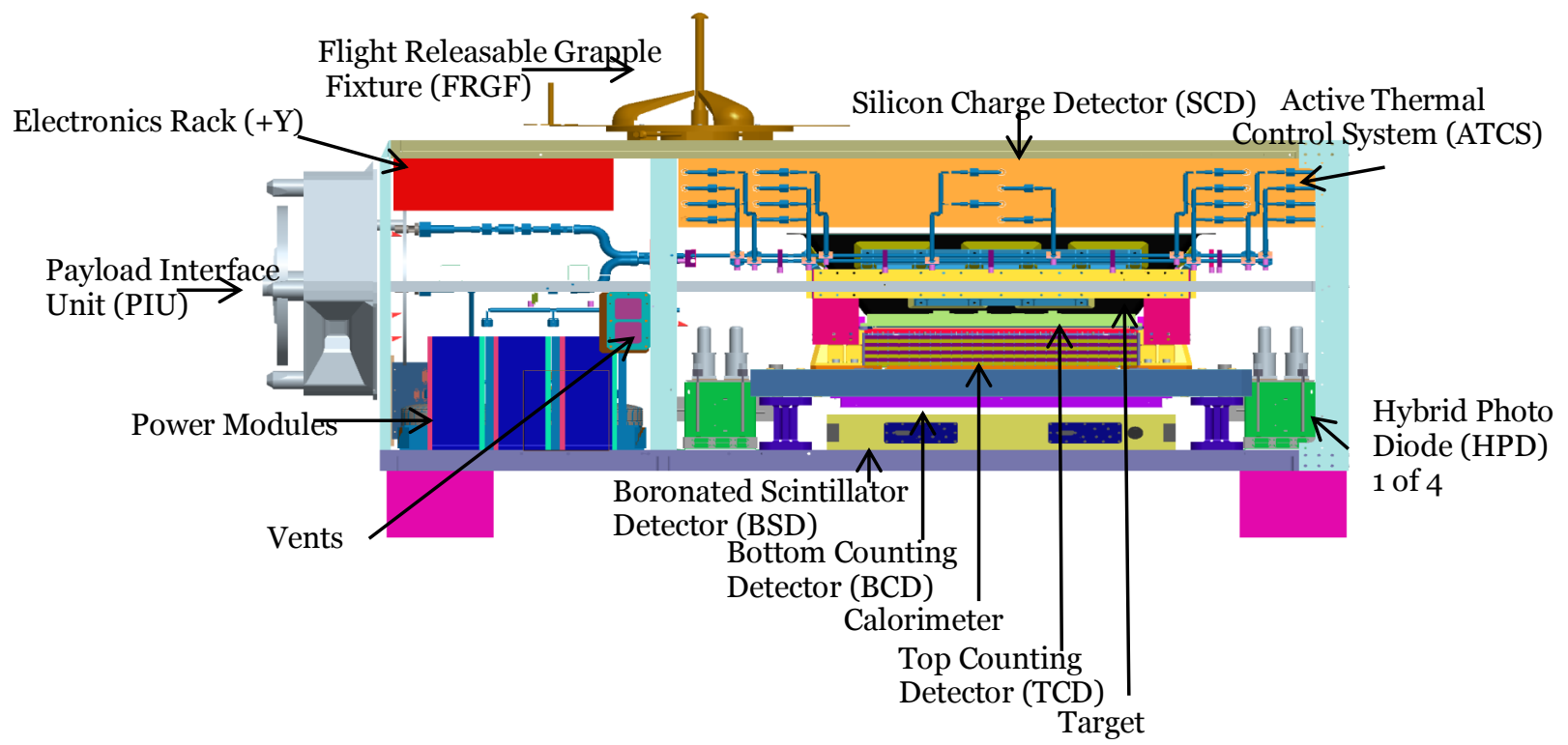

Fig.1. The ISS-CREAM payload configuration 


\section{Flight Operations and Data Handling}

After launch, flight operations were managed at the SOC at University of Maryland (UMD) by ISS-CREAM collaboration members, in coordination with the Payload Operations Integration Center (POIC) at Marshall Space Flight Center (MSFC). Operators staff the SOC 24/7 to command the instrument, receive and monitor data and instrument performance, as well as back-up and archive data on additional UMD servers and distribute data to collaboration sites. Figure 2 shows the flight data that were displayed for web monitoring. The facilities and processes at UMD are the same that have been used for the Antarctic balloon flights. There is 32 GB of onboard storage system with four-fold redundancy (128 GB total) to handle and store data on a single drive for up to 24 days in case of prolonged loss of signal. ISS-CREAM has a continuous downlink of housekeeping and science data in near real-time. Commanding to adjust operational parameters was done periodically as needed. After ISS-CREAM was installed on the JEM-EF, the instrument parameters were optimized within the first two weeks and nominal data collection and operations were continuously run until flight termination. Calibration and science data are processed into the CERN ROOT format and divided into levels called L0, L1, and L2. L0 is the first level used to analyze detector performance and determine in-flight calibration parameters. It consists of raw instrument signals with pedestals subtracted. Calibrations and channel mappings are then applied to the L0 data in order to generate L1 data that consists of signals in physics units such as energy and charge. The final level of processing for data analysis is the reconstruction of incident particle energy, charge, and trajectory into the L2 dataset. Each level of the processing and data is shared between collaboration institutes. This method of data processing, reconstruction, and distribution has been used by the CREAM collaboration for the Antarctic balloon flights. The flight phase and individual operation flow including instrument power, thermal, command and data for the pre- and post-launch are shown in Figure 3.
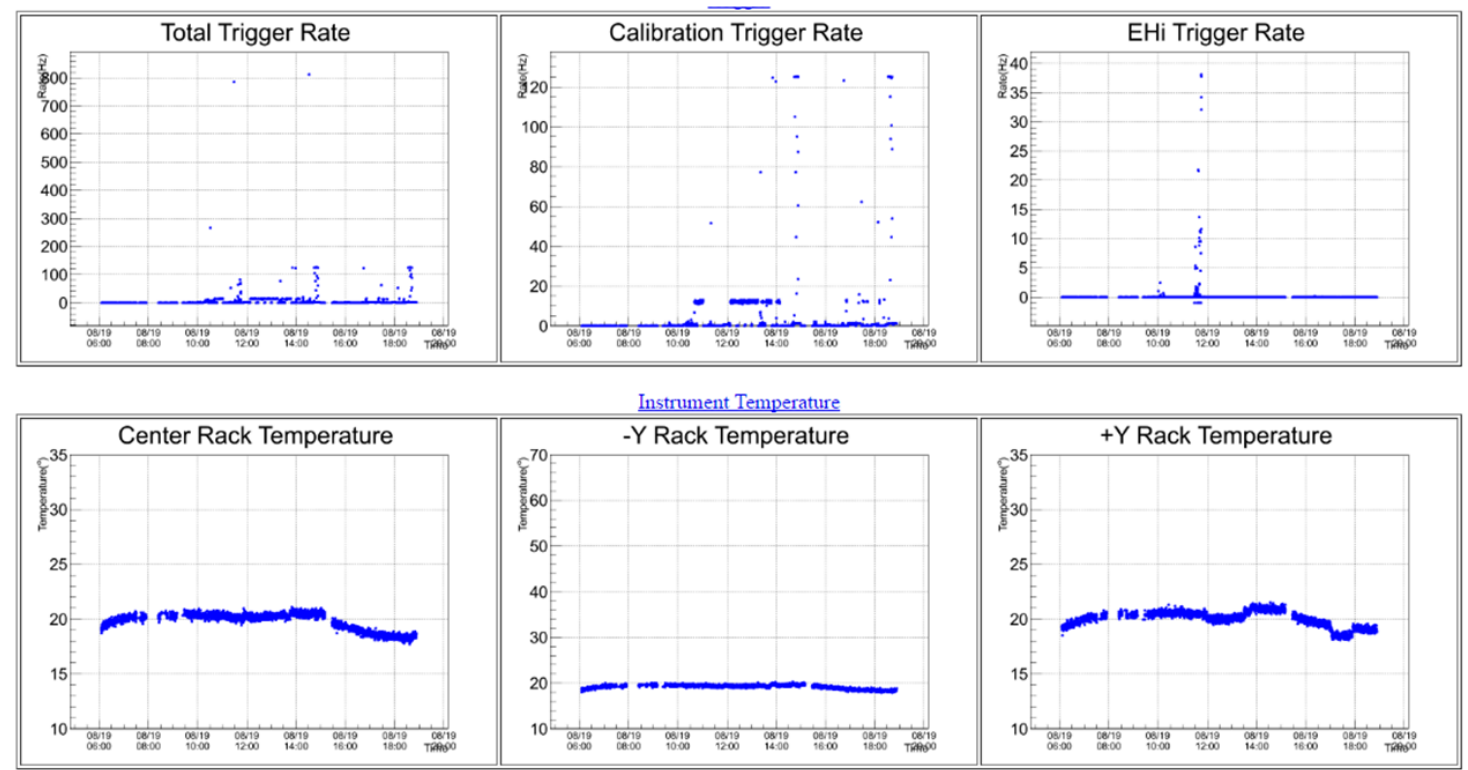

Fig.2. Web monitoring data 


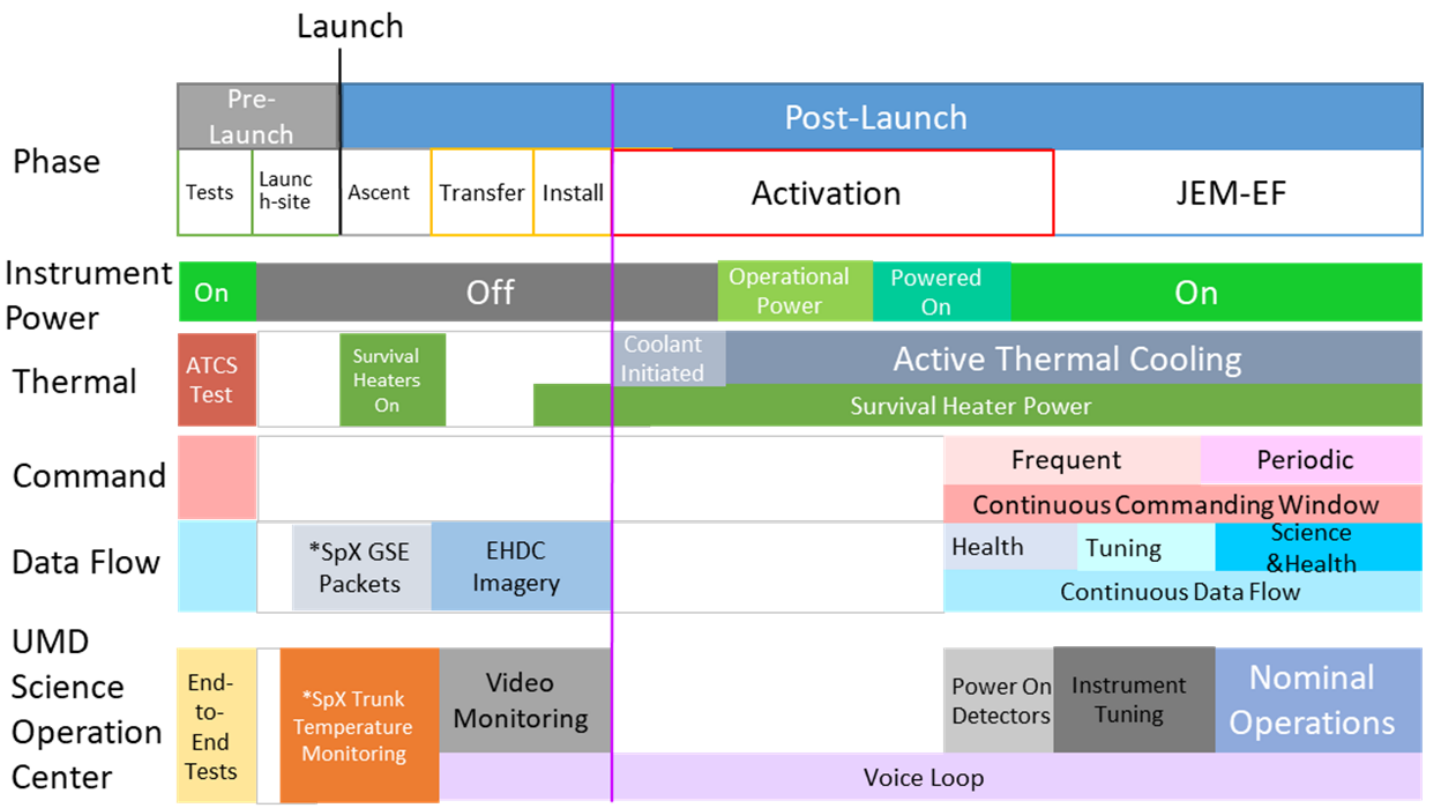

Fig. 3. Operation flow. This shows the flight phase and individual operation flow for the pre- and post-launch time frames.

\section{Communication for data and command}

The ISS-CREAM operation is the same as CREAM, except the Huntsville Operations Support Center (HOSC) and POIC. The instrument interface with the ISS is Ethernet based, as was the Command and Data handling Module for the CREAM balloon flights. The CREAM DAQ (CDAQ) has been modified to interface with the POIC using the Telescience Resources Kit (TReK http://trek.msfc.nasa.gov). A significant rewrite of software was avoided through use of the Software Toolkit for Ethernet Lab-Like Architecture (STELLA) developed by the Boeing Company. The CDAQ handles command and data both in real time and in playback. The command uplink and data downlink are through the Tracking and Data Relay Satellite System (TDRSS), exactly analogous to CREAM balloon flight operations. The CREAM team has extensive experience in running the SOC at UMD for 7 balloon flights over a period of 7 years. The SOC has been configured to provide secure data links with the POIC; adequate data processing hardware; and the data archive. As the raw data sets are piped to the SOC, backup copies are made immediately and stored on RAID system in the UMD Research Data Center (RDC). The existing Internet bandwidth between MSFC and the SOC averages $4 \mathrm{Mbps}$, which is sufficient to transmit the data. The necessary operating systems and software, including CDAQ and STELLA, were installed on the server system, SFC, and client computers for the ground operations. Figure 4 shows the data flow from the payload to SOC and command flow from SOC to ISS-CREAM. 

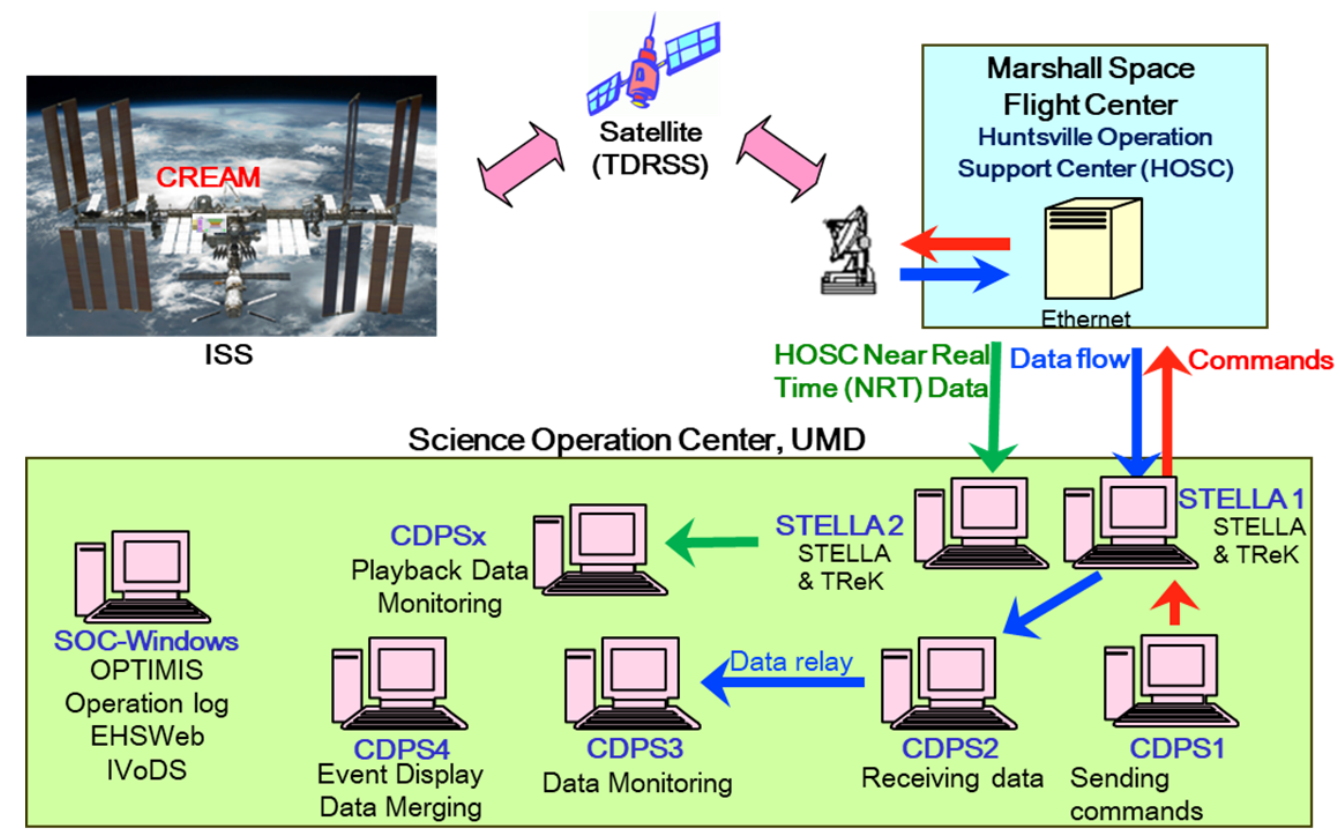

Fig. 4. Data and command flow. This shows the data flow from ISS-CREAM to SOC and command flow from SOC to ISS-CREAM.

\section{Science Flight Computer}

The SFC is central to the data-acquisition system. It consists of a redundant pair of flight computers (SFC-A and SFC-B), where one is powered and the other is a spare. Each flight computer consists of a commercial off-the-shelf single-board computer and USB host controller board (USB-HCB). The USB-HCB contains the USB host controller, one EIDE boot-source flash drive (boot- 0 ), and three USB boot-source flash drives (boot-1, -2, -3). Only one boot-source is powered at a time with the other three as cold spares. The USB-HCB is the main USB interface of the SFC and connects directly to the SFC-interconnect (SFC-IC) and instrument-interface unit (IIU). Also on each SFC-IC card there are four USB flash drives, independent of the boot-source flash drives, for on-board data storage. The purpose of the IIU is to autonomously monitor the functionality of the SFC and take corrective actions if necessary. Management of these functions is performed by a logic programmed onto the IIU FPGA through the use of two watchdog timer (WDT) conditions called local-USB WDT and ground WDT. The local-USB WDT is a ten-minute timer that receives a periodic response initiated by the ISS. The ISS-CREAM data-acquisition system runs on the SFC (CDAQ server). The ground WDT is a three-hour timer that receives a periodic response by ground-based data-acquisition systems (CDAQ client) via command. Each WDT is reset independently by its respective source. ISS systems are also able to send CDAQ commands to ISS-CREAM through a timed commandable sequence called a time-liner.

\section{Summary}

The ISS-CREAM instrument was launched in August, 2017 and has been operated for about one and half years successfully. After installation to ISS, SOC managed the operations 24/7 to command the instrument, receive and monitor data and instrument performance, as well as 
back-up and archive data. The CDAQ interface with the POIC using the Telescience Resources Kit and handles command and data both in real time and in playback. The command uplink and data downlink are through the TDRSS, exactly analogous to CREAM balloon flight operations. The performance of SFC, communication system, data, command flow, SOC operation are all successful as expected during the flight.

\section{Acknowledgments}

This work was supported in the U.S. by NASA grant NNX17AB41G, in Korea by National Research Foundation grants 2018R1A2A1A05022685 and 2018R1A6A1A06024970, and their predecessor grants. It was also supported in France by IN2P3/CNRS and CNES and in Mexico by DGAPA-UNAM project IN109617. The authors thank NASA GSFC WFF and its contractors for engineering support and project management, JSC ISS Program Office for the launch support and ISS accommodation, MSFC for the operational support, and KSC and SpaceX for the launch support.

\section{References}

[1] E.S. Seo et al., Cosmic Ray Energetics And Mass for the International Space Station (ISS-CREAM), Adv. Sp. Res. 53, 10, 1451-1455 (2014)

[2] E.S. Seo et al., Cosmic-ray energetics and mass (CREAM) balloon project, Adv. In Sp. Res. 33.10, $1777-1785$ (2004)

[3] I.H. Park et al., Silicon charge detector for the CREAM experiment, Nucl. Inst. Meth. A 570, 286291 (2007)

[4] J. Lee et al., Design, Fabrication and Performance of the Silicon Charge Detector for the ISSCREAM, Proc. 34th Int. Cosmic Ray Conf., The Hague, Netherlands, ID 693 (2015)

[5] Y.S. Hwang et al., Construction and Testing of a Top Counting Detector and a Bottom Counting Detector for the Cosmic Ray Energetics And Mass Experiment on the International Space Station, J. INST 10 (07), P07018 (2015)

[6] H.J. Hyun et al., Performances of photodiode detectors for top and bottom counting detectors of ISS-CREAM experiment, Nucl. Inst. Meth. A 787, 134-139 (2015)

[7] J.T. Link et al., Design and Performance of the ISS-CREAM Boronated Scintillator Detector, Proc. 34th Int. Cosmic Ray Conf., The Hague, Netherlands, ID 611 (2015)

[8] N. Picot-Clemente et al., Performance of the ISS-CREAM Calorimeter, Proc. $35^{\text {th }}$ Int. Cosmic Ray Conf., Busan, Korea, 247 (2017) 